\title{
Commentary: Barriers to concordance with antidiabetic drugs-cultural differences or human nature?
}

Trisha Greenhalgh

University College London, London N19 5LW

Trisha Greenhalgh professor of primary health care

p.greenhalgh@ pcps.ucl.ac.uk
British Indian and British Pakistani patients think that the products of the pharmaceutical industry are potent and may do harm. They do not want to take any more tablets than they absolutely have to. They discontinue their tablets if they have major side effects. They distrust doctors who stand to gain financially from prescribing particular drugs. They use creative strategies to titrate the dose of their drugs to the lowest that they consider works. ${ }^{1}$ In all these respects, the sample reported here has more similarities to than differences from the indigenous British population, and indeed most other groups studied in relation to medicine taking. ${ }^{2}$

That said, this study is no less important than it would have been if the authors had detected a set of perceptions and attitudes that were unique to British South Asians. In terms of action points for practice and policy, however, I disagree with these authors that the way forward is to focus primarily on cultural differences. Instead, I would have preferred them to link their findings more closely with the extensive evidence base on medicine concordance, defined as "agreement between the patient and the health care professional, reached after negotiation, that respects the beliefs and wishes of the patient in determining whether, when and how their medicine is taken, and the primacy of the patient's decision [is recognised]."

Key prerequisites for concordance are: (a) a power sharing consulting style, in which the clinician engages with, acknowledges, and gives appropriate weight to the patient's values and goals; (b) open discussion of all options, which requires explicit seeking out of the patient's perspective rather than second guessing; (c) adequate information for decision making, which in turn depends on both information sharing by the clinician and adequate health literacy in the patient, as well as an understanding by the clinician of the beliefs and perceptions that are driving the patient's choices; (d) a fair balance of discussion (with the clinician doing as much listening as talking); and (e) adequate time. ${ }^{2-4}$

Of course, when caring for patients across a cultural divide, especially when the patient has limited proficiency in English, many of these prerequisites are harder to achieve. Lawton et al rightly emphasise the key role of the bilingual health advocate in this regard, but it is important to note that this role is not merely to translate instructions from doctor to patient but to explain the patient's perspective (and encourage the doctor to take it on board). The patient's level of health literacy-that is, his or her capacity to obtain, interpret, and understand information about health and healthcare services, in any language-may be more important than "culture" in explaining beliefs about drugs that are highly discordant with those of Western healthcare professionals. ${ }^{4}$

As Lawton et al imply, a high policy priority should be to develop effective education programmes that tackle basic ignorance about the nature, course, and management of diabetes, and to deliver these in the full range of ethnic languages. In the words of UK secretary of state John Reid, "The more capable everyone is of understanding the issues that relate to their own health, the more they will be able to take responsibility for their own health-whether taking the right course of medication or knowing what a good diet is."

Competing interests: None declared.

1 Lawton J, Ahmad N, Hallowell N, Hanna L, Douglas M. Perceptions and experiences of taking oral hypoglycaemic agents among people of Pakistani and Indian origin: qualitative study. BMJ 2005;330:1247-9.

2 Marinker M, Blenkinsopp A, Bond C. From compliance to concordance: achieving shared goals in medicine taking. London: Roval Pharmaceutical achieving shared goals in medicine
Society of Great Britain, 1997.

3 Britten N, Stevenson F, Gafaranga J, Barry C, Bradley C. The expression of aversion to medicines in general practice consultations. Soc Sci Med 2004;59:1495-503

4 Stevenson FA, Cox K, Britten N, Dundar Y. A systematic review of the research on communication between patients and health care professionals about medicines: the consequences for concordance. Health Expect 2004;7:235-45.

5 National Consumer Council. Health literacy-being able to make the most of health. London: National Consumer Council, 2004.

doi 10.1136/bmj.38464.595741.AE

This article was posted on bmj.com on 9 May 2005: http://bmj.com/cgi/ doi/10.1136/bmj.38464.595741.AE

\section{One hundred years ago}

\section{The treatment of inebriety}

Mr. SHEIL, who has just retired after twenty-five years' service as a metropolitan police magistrate, made some interesting observations on inebriety in the course of an interview with a representative of the Evening Standard on December 27th, 1904. As far as his district-that of the Westminster Police Court-was concerned, he could not say that he had noted any improvement in the point of temperance. The men, he thought, were as bad, and the women worse, than when he first took charge of it. Temperance in the sense of moderation was of no avail to the confirmed drunkard, because it was impossible to him, while as for total abstinence, although perhaps it might endure for a year, it usually was but a thing of a day. In almost every case a drink offered in a temperate and friendly spirit led to a fresh outbreak, and matters were then as bad or worse than before. Mr. Sheil, we believe, never thought it worth while to make use of the discretionary powers as to black listing granted him under the Act of 1902; and this fact, coupled with the views which he expressed, is justification for believing that he would be on the side of those who contend that none of the Acts at present in force with regard to inebriety really meet the situation. (BMJ 1905;i:32) 